\title{
Experimental analysis on the dynamic wake of an actuator disc undergoing transient loads
}

\author{
W. Yu' ${ }^{1} \cdot$ V. W. Hong ${ }^{1}$ C. Ferreira ${ }^{1}$ G. A. M. van Kuik ${ }^{1}$
}

Received: 7 April 2017 / Revised: 10 September 2017 / Accepted: 11 September 2017 / Published online: 26 September 2017

(C) The Author(s) 2017. This article is an open access publication

\begin{abstract}
The Blade Element Momentum model, which is based on the actuator disc theory, is still the model most used for the design of open rotors. Although derived from steady cases with a fully developed wake, this approach is also applied to unsteady cases, with additional engineering corrections. This work aims to study the impact of an unsteady loading on the wake of an actuator disc. The load and flow of an actuator disc are measured in the Open Jet Facility wind tunnel of Delft University of Technology, for steady and unsteady cases. The velocity and turbulence profiles are characterized in three regions: the inner wake region, the shear layer region and the region outside the wake. For unsteady load cases, the measured velocity field shows a hysteresis effect in relation to the loading, showing differences between the cases when loading is increased and loading is decreased. The flow field also shows a transient response to the step change in loading, with either an overshoot or undershoot of the velocity in relation to the steady-state velocity. In general, a smaller reduced ramp time results in a faster velocity transient, and in turn a larger amplitude of overshoot or undershoot. Time constants analysis shows that the flow reaches the new steady-state slower for load increase than for load decrease; the time constants outside the wake are generally larger than at other radial locations for a given downstream plane; the time constants of measured velocity in the wake show radial dependence. The data are relevant for the validation of numerical models
\end{abstract}

W. Yu

W.Yu@tudelft.nl

Faculty of Aerospace Engineering, Delft University of Technology, Kluyverweg 1, 2629 HS Delft, The Netherlands for unsteady actuator discs and wind turbines, and are made available in an open source database (see Appendix).

\section{List of symbols}

A Area of the disc $\left(\mathrm{m}^{2}\right)$

$A_{\mathrm{w}} \quad$ Area of stream-tube section in the wake $\left(\mathrm{m}^{2}\right)$

$C_{\mathrm{t}} \quad$ Thrust coefficient (-)

$d \quad$ Displacement of the moving disc (m)

$D \quad$ Diameter of the disc (m)

$p_{0} \quad$ Pressure at the far upstream $\left(\mathrm{N} \mathrm{m}^{-2}\right)$

$p_{\mathrm{w}}$ Pressure in the wake $\left(\mathrm{N} \mathrm{m}^{-2}\right)$

$\delta t^{*} \quad$ Reduced ramp time $\left(\delta t^{*}=\frac{\delta t}{D / V_{0}}\right)(-)$

$t \quad$ Time (s)

$\tau_{0} \quad$ Time scale of dynamic inflow $\left(\frac{D}{V_{0}}\right)(\mathrm{s})$

$\tau^{*} \quad$ Time constant of velocity decay in the wake (s)

$V_{0} \quad$ Free stream velocity $\left(\mathrm{m} \mathrm{s}^{-1}\right)$

$V_{\mathrm{d}} \quad$ Velocity at the disc $\left(\mathrm{m} \mathrm{s}^{-1}\right)$

$V_{\mathrm{w}} \quad$ Velocity in the wake $\left(\mathrm{m} \mathrm{s}^{-1}\right)$

$V_{x} \quad$ Axial velocity $\left(\mathrm{m} \mathrm{s}^{-1}\right)$

\section{Introduction}

Momentum Theory is the basis of Blade Element Momentum theory (BEM), the currently most popular design theory for rotor aerodynamics. However, the equilibrium state assumption of momentum theory is challenged by, for example, the unsteady loads on wind turbines, which is determined by the unsteady wind and control. These unsteady wind and load effects include turbulence, wind shear, motion of the blade, and the passive and active aerodynamic control strategies, such as yaw, pitch control and smart rotor control, where a wind turbine operates in a dynamic state. An unsteady rotor/actuator loading results in an unsteady 
wake flow and induction field, commonly named 'Dynamic Inflow'.

A number of experimental studies were conducted focusing on the the dynamic load measurements of a wind turbine under practical situations, such as a pitch angle transient, yaw and wind speed change etc. The delay of response of flapwise moment and power from a change of pitch angle on a Nibe B HAWT was first observed by Øye (1986). In the project JOULE I (Snel and Schepers 1995), both axisymmetric and yawed cases were tested. Two turbines were used for the test. The measurement on the full scale turbineTjæreborg turbine focused on pitching transient and yawed flow conditions (Øye 1991a, b). The measurement on a wind tunnel turbine tested in the TUDelft open jet wind tunnel focused on the wind gust and yawed flow. The velocity at three fixed positions downstream the rotor plane was measured by a hot wire for some particular test cases. However, the wind gust was not simulated successfully because the generation of gust were slower than the dynamic inflow time scale. The velocity measured downstream planes was not representative as the dynamics was azimuthal position dependent in this set-up. In the NREL/NASA Ames wind tunnel test (Hand et al. 2001), a series of operational conditions ranging from upwind axial operation, pitch step, yaw operation and variation in AOA were tested. The most important improvement of these tests on dynamic inflow was that the instantaneous pressure at five radial positions instead of only blade bending moment in previous projects were measured. This provides the information for studying the radial dependency of the time constant along the blade (Schepers 2007).

However, no experimental work using an actuator disc to investigate the unsteady flow field of an open rotor has been reported. The use of an actuator disc to investigate the dynamic inflow effects is of interest from two fundamental perspectives. First, it is the basis of the Blade Element Momentum theory. It lends a fundamental perspective to many existing engineering dynamic inflow models, which are also derived from actuator disc theory. This is the case for the commonly used engineering dynamic inflow models of Pitt and Peters (1981), Øye (Snel and Schepers 1995; Øye 1990) and ECN (Schepers 2012). Second, the use of actuator disc model can help the study dedicated to basic problem by getting independent from the effect of blade geometry, rotational momentum, tip vortices etc.

The concept of actuator disc, on which the Momentum Theory is based, was introduced by Froude (1889), where the rotor plane is treated as a disc representing a rotor with an infinite number of blades. Porous discs were used by many researchers to physically represent actuator discs experimentally, different types of porous discs were tried out (Sforza et al. 1981; Pierella and Sætran 2010; Medici 2005; Aubrun et al. 2011; Lignarolo et al. 2014;
Muller et al. 2015). Castro (1971) discovered a reverse flow region dominating the wake of a perforated plate at low values of porosity, and the vortex street was not observed for high values of porosity. The wake properties of a wind turbine model and a porous disc model was compared experimentally by Aubrun et al. (2013), which show indistinguishable difference in their wake after $3 D$ downstream distance. The pressure recovery was shown at $1.5 \mathrm{D}$ for both models in a neutral atmospheric boundary layer with a IT $=13 \%$ at the disc hub for $C_{\mathrm{t}}=0.5$. A good match in the basic properties, such as coefficient of thrust and energy, field of velocity, pressure and enthalpy was observed between a wind turbine and an actuator disc model using PIV measurements by Lignarolo et al. (2016), which suggests that the near wake of a wind turbine can be also represented by the near wake of a porous disc. However, the wake was shown not fully expanded at the distance of $2.2 \mathrm{D}$ downstream for the two models with $C_{\mathrm{t}}=0.93$ in this low turbulence condition. Chamorro and Porté-Agel (2009) found that the boundary-layer turbulence has an important effect on the wake development, which was found to even persist at $x / D=15$. Not only a single turbine was represented by porous disc, the wind turbine array in a wind farm can also be imitated by porous discs. The mean kinetic energy transport within the wake of an array of rotating wind turbine and an array of static porous discs was compared experimentally by Camp and $\mathrm{Cal}$ (2016). The spatio-temporal characteristics of a model wind farm consist of 100 porous discs was studied by Bossuyt et al. (2017).

In addition to the usage of an actuator disc model in aforementioned experiments for steady load cases, it was also used in some unsteady cases. The characteristics of instantaneous thrust of an impulsively started porous surface was studied experimentally in a water tunnel and numerically using a point vortex wake model by Johnson et al. (2013); however, this study did not analyse the wake. Furthermore, the disc had relative motions to the incoming flow in this set-up. The relative motion between the disc and its instantaneous wake results in a temporal impulse exerted by the disc on the fluid. Flow in such situations is different from that in a general dynamic inflow problem.

In this study, a novel approach is used to experimentally investigate the basic problem of wake flow response of an actuator disc undergoing unsteady loads using a disc model with variable porosity. The unsteady load is generated by a ramp type variation of porosity of the disc, at several reduced times of the ramp motion. The unsteady loads on the actuator disc are measured with a load cell. The flow downstream of the disc (in and outside of the wake) is measured at various positions using a hot-wire anemometer. The results create a database for the validation of unsteady numerical models, in prediction of the dynamic induction in the near 
wake $(0.5 D-3 D)$ of an actuator disc or a rotor, to more accurately predict load and induction on the disc itself under unsteadiness.

\section{Experimental methods}

This section provides information on the experimental methods used in this paper, including the description of the experimental set-up, the wind tunnel, the unsteady actuator disc model, the load and flow measuring techniques, tested load cases and the data analysis method. A photograph and a schematic representation of the set-up is shown in Fig. 1. The tower is mounted directly on the external OJF balance which is equipped with six load cells. The hot-wire is mounted on a traverse system, which can traverse axially ( $x$ direction) and radially ( $y$ direction) the probe to the measuring points.

\subsection{Wind-tunnel and actuator disc model}

The experiments are carried out in the low speed closedcircuit Open Jet Facility (OJF) of Delft University of Technology. The OJF wind tunnel has an octagonal cross-section of $2.85 \times 2.85 \mathrm{~m}^{2}$ and a contraction ratio of $3: 1$, it is free to expand in an area of $13.7 \times 6.6 \times 8.2 \mathrm{~m}^{3}$. The free stream velocity ranges from 3 to $34 \mathrm{~m} \mathrm{~s}^{-1}$ with a flow uniformity of $0.5 \%$ and a turbulence level of $0.24 \%$, powered by a $500 \mathrm{~kW}$ electric motor. The temperature in the test section is maintained at a constant temperature of $20^{\circ} \mathrm{C}$ by a $350 \mathrm{~kW}$ heat exchanger through the experiments.

The purpose of the experiment is to investigate the unsteady load effects: the load on the disc is varied through adjustment of the relative open area (porosity) formed by two identical parallel porous discs. With the aim of reaching the optimal thrust coefficient of a wind turbine around 8/9 and a distinct load change can be achieved with a small displacement of the disc, different types of discs were tried out (Hong 2015). The final chosen discs are made from $2.0 \mathrm{~mm}$ thick aluminium plate with punctured $10 \times 10 \mathrm{~mm}^{2}$ square holes. Each disc has a diameter of $600 \mathrm{~mm}$, hole-to-hole spacing of $2.0 \mathrm{~mm}$, and porosity of $69.4 \%$. The characteristics are summarized in Table 1. The uniform distributed porosity over the disc is aimed for uniform distributed load. The magnitude and rate of porosity change are defined by the displacement and speed of the moving disc, respectively. The gap clearance between the two discs is kept at around $1.7 \% \circ D$, which is the optimized gap at this set-up for the tested wind speed. Ideally the smaller the gap, the closer it can represent one single actuator disc model. However, if the two discs are tightly pressed when wind is on, the large friction between the two discs will affect the accuracy of disc displacement. The high and low porosity states of the actuator disc model are shown schematically in Fig. 2.

The nacelle and actuator system is shown in Fig. 3. As seen, a linear slider is mounted inside the channel-type nacelle. The fixed disc is mounted directly on the top of

Table 1 Characteristics of tested porosity disc

\begin{tabular}{ll}
\hline Material & Aluminium plate \\
Diameter $(\mathrm{mm})$ & 600 \\
Hole shape & Square \\
Hole size $(\mathrm{mm})$ & $10 \times 10 \mathrm{~mm}^{2}$ \\
Hole-to-hole space $(\mathrm{mm})$ & 2.0 \\
Thickness $(\mathrm{mm})$ & 2.0 \\
Porosity $(\%)$ & 69.4 \\
\hline
\end{tabular}
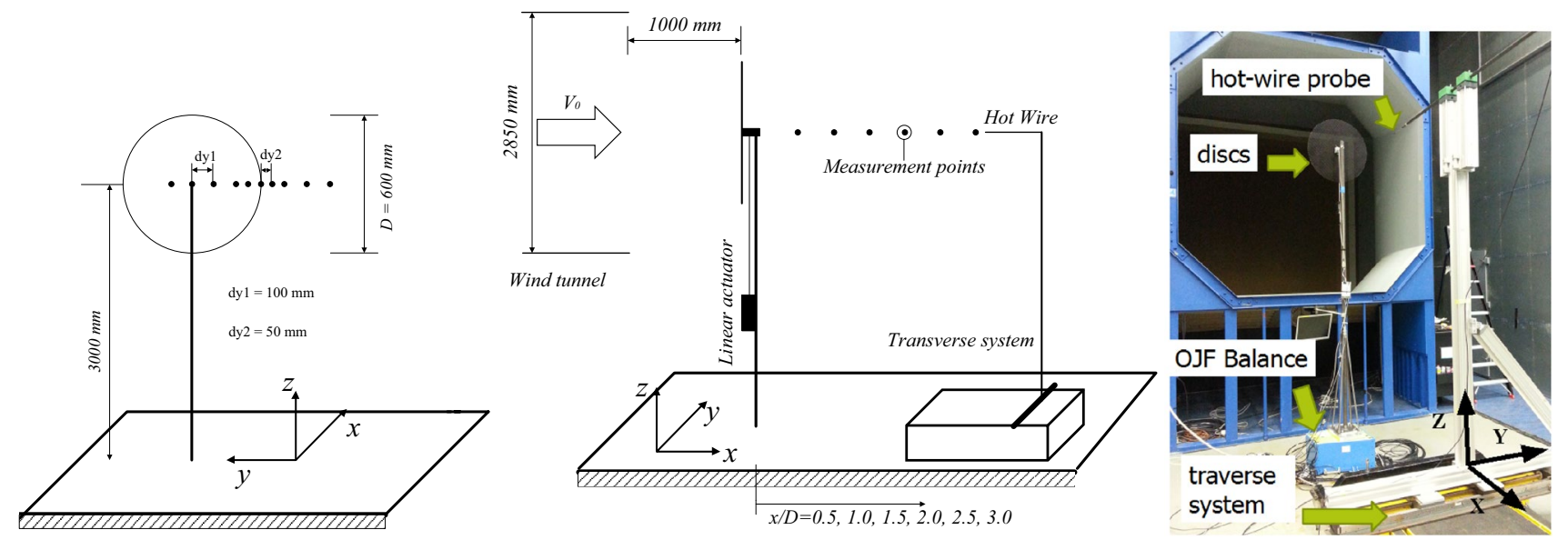

Fig. 1 Schematic of the set-up (only for reference, not to scale), front view (left), side view (middle) and photograph of the test section with the actuator disc model (right) 


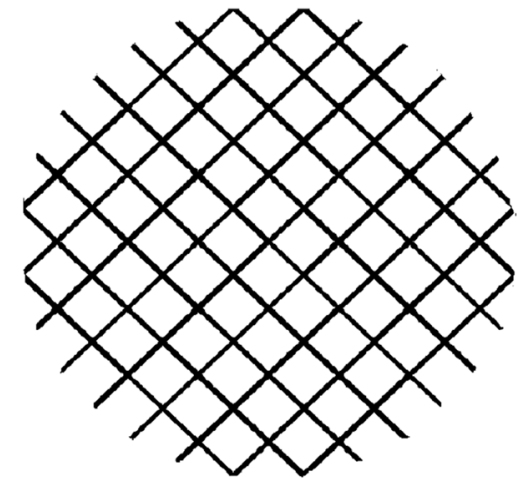

(a) porosity at $69.4 \%$

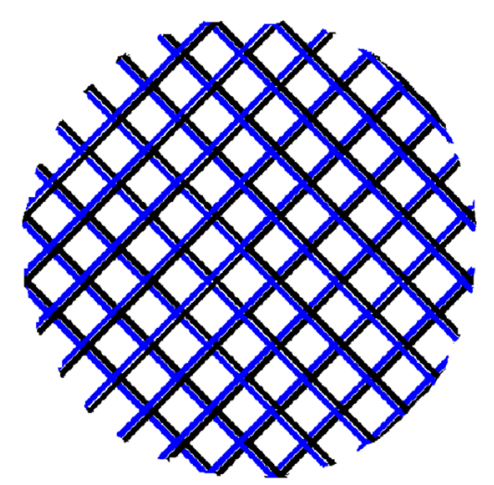

(b) porosity at $44.4 \%$

Fig. 2 Schematically representation of the actuator disc model at high and low porosity states

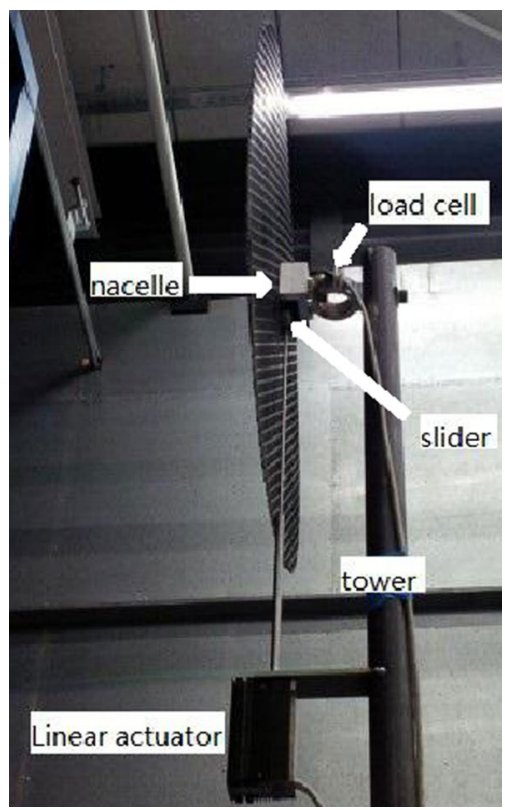

Fig. 3 Nacelle system and actuate scheme the nacelle, the moving disc is mounted on the moving rail of the slider. The clearance between the two discs can be adjusted by shims.

The slider is connected to a linear voice coil actuator SMAC LCA31-012-62CS through a rod. The moving disc together with the moving rail of the slider is actuated vertically by the linear actuator. The copper coil rides inside a magnet assembly, in which way the reaction force can be controlled by current flows through the coil. The movement of the disc is controlled by a SMAC LAC-1 controller. The actuator is equipped with a resolution of $0.1 \mu \mathrm{m}$, and the sampling intervals of the controller is up to $0.2 \mathrm{~ms}(5 \mathrm{~ms}$ is used in the experiment). The entire nacelle system is mounted to the tower through a TUD-made sensor, which is a uni-axial load cell.

\subsection{Test cases and data averaging}

The tested wind speed for this experiment is set at $6 \mathrm{~m} \mathrm{~s}^{-1}$, which gives a diameter based Reynolds number of 270,680. Knight (1926) discovered that the aerodynamic thrust of an actuator disc is insensitive to Reynolds number when it is larger than 150,000 using tunnel test of three different types of discs. Hoerner (1965) summarized that above diameterbased Reynolds number of 1000, the drag coefficient of discs (and other plates) is practically constant up to the highest tested Reynolds number of $10^{7}$. Besides the consideration of Re number, the upstream velocity is chosen based on a balance of a distinct force change for the given porosity change and an acceptable axial force between the two discs. The control of the actuator motion and data acquisition are synchronized in the LabVIEW program. The output signals of the hot-wire, the load cell, the OJF balance and the position of motor shaft are sampled at $2 \mathrm{kHz}$.

There are two steady thrust coefficient $\left(C_{\mathrm{t}}\right)$ corresponding to the two porosity states in Fig. 2, henceforth, called $C_{\mathrm{t}}^{\text {low }}$ and $C_{t}^{\text {high }}$ accordingly. The velocity aft of the disc under the steady states is measured as a reference. To represent pitching transients of a wind turbine, a ramp change profile is prescribed in all unsteady cases (see Fig. 4), with $\delta t$ representing the ramp time. The reduced ramp time $\left(\delta t^{*}\right)$ is used in this experiment, which is defined by Eq. 1 .

$\delta t^{*}=\frac{\delta t}{D / V_{0}}$

As known from the experiments in project JOULE I and II (Snel and Schepers 1995; Schepers and Snel 1995), the time scale in the dynamic inflow process appeared to be on the order of $0.3-0.5 \frac{D}{V_{0}}$. Therefore, three different reduced ramp times $\delta t^{*}=0.2,0.4,0.8$ are tested in this experiment. The steady and unsteady tested cases are summarized in Table 2. 


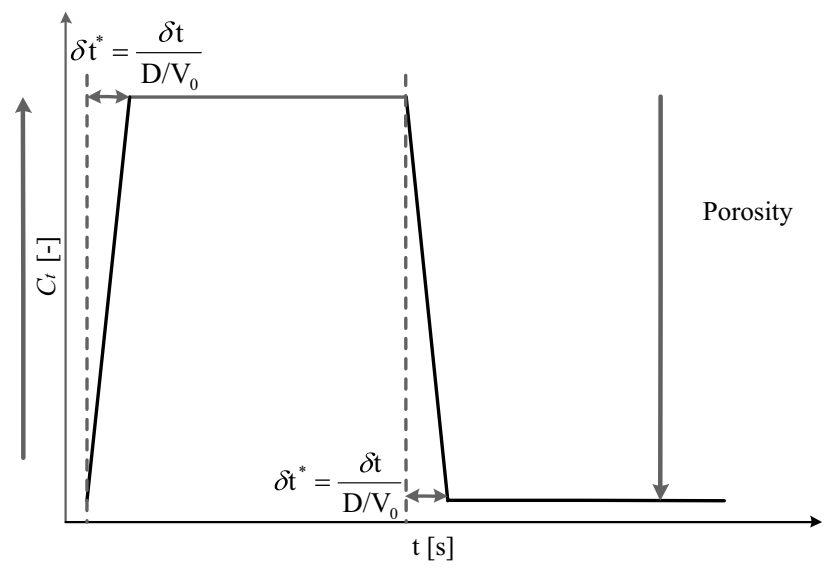

Fig. 4 Unsteady load profile

Table 2 Tested steady and unsteady cases

\begin{tabular}{lllll}
\hline Cases & Number & Porosity $(\%)$ & Thrust & $\delta t^{*}$ \\
\hline Steady cases & SI & 69.4 & $C_{\mathrm{t}}^{\text {low }}$ & - \\
& SII & 44.4 & $C_{\mathrm{t}}^{\text {high }}$ & - \\
Unsteady cases & UI & $44.4 \rightarrow 69.4$ & $C_{\mathrm{t}}^{\text {high }} \rightarrow C_{\mathrm{t}}^{\text {low }}$ & 0.2 \\
& UII & $44.4 \rightarrow 69.4$ & $C_{\mathrm{t}}^{\text {high }} \rightarrow C_{\mathrm{t}}^{\text {low }}$ & 0.4 \\
& UIII & $44.4 \rightarrow 69.4$ & $C_{\mathrm{t}}^{\text {high }} \rightarrow C_{\mathrm{t}}^{\text {low }}$ & 0.8 \\
& & & & \\
\hline
\end{tabular}

The acquiring period for steady cases is $20 \mathrm{~s}$, the mean velocity and thrust is directly time-averaged. For the unsteady experimental cases, unsteady load is varied by dynamically changing the porosity of the disc. Factors as turbulence and signal noise will affect the instantaneous results. To obtain the ensemble average velocity profile, multiple unsteady load cycles for the same measuring point (x) at the same test conditions are measured consecutively. The instantaneous value is denoted as $u_{i}(\mathbf{x}, t)$, with a number of experimental cycles at the identical point $u_{i}^{(n)}(\mathbf{x}, t)$, the ensemble average value $U_{i}(\mathbf{x}, t)$ is then defined by Eq. 2 , where $n$ is the cycle index and $N$ is the total number of cycles.

$U_{i}(\mathbf{x}, t)=\frac{1}{N} \sum_{n=1}^{N} u_{i}^{(n)}(\mathbf{x}, t)$

For this experiment, $N$ is 10 , the reason behind this choice is given in Sect. 2.5.

\subsection{Load measurement and processing}

Initially, the OJF balance in Fig. 1 is used for load measurement. In addition to that, to minimise the influence of the dynamics of the supporting structures, a load cell (as shown in Fig. 3) is installed at the disc axis, which is capable sensing uni-axial loads up to $500 \mathrm{~N}$. The force measured by the load cell are eventually used for all unsteady cases.

Tests at the absence of any wind $\left(V_{0}=0\right)$ and at the designed wind speed $\left(V_{0}=6 \mathrm{~m} \mathrm{~s}^{-1}\right)$ while the actuator is activated are carried out to determine the frequency response of the load cell systems. Figure 5a, c presents the thrust coefficient obtained by the load cell and its power spectrum during the unsteady load cycle, while the wind tunnel is off. The measured $C_{\mathrm{t}}$ and its power spectrum with the wind tunnel switched on are shown in Fig. 5b, d. When the wind tunnel is off and the actuator is activated, the load is purely inertial force of the system vibration. From the power spectrum, the natural frequency of the system mechanical vibration is around $5.5 \mathrm{~Hz}$. When the wind is on, Fig. $5 \mathrm{~b}$ shows a distinct step change in aerodynamic force. However, the natural frequency is evident in $C_{\mathrm{t}}$ in Fig. $5 \mathrm{~b}$ and in its spectrum Fig. $5 \mathrm{~d}$. The high energy at low frequency for the configuration with wind in Fig. 5d is due to the presence of the two states in the signal, which is not a subject of this article.

Based on the system study, a 6th-order Butterworth bandstop filter with a normalized edge frequencies of 4 and $8 \mathrm{~Hz}$ is designed to filter out the effect of system vibration, and a cut-off frequency of $10 \mathrm{~Hz}$ is applied. The filtered $C_{\mathrm{t}}$ is also shown in Fig. 5b. As seen, the filtered data can capture the trend of the $C_{\mathrm{t}}$ well.

\subsection{Velocity measurement}

A constant temperature thermal anemometer system of TSI IFA 300 is used to measure the axial velocity. Calibration of the hot-wire anemometer is done at the beginning of each measurement, using a TSI Model 1127 Manual Velocity Calibrator.

As shown in Fig. 1, the velocity field at planes of $0.5 D$ to $3.0 D$ with an interval of $0.5 D$ is measured. A spatial interval of $0.167 D$ is taken between measurement points in the radial direction. In the region of $y / D=\frac{1}{3}-\frac{2}{3}$, the resolution is increased to $0.083 \mathrm{D}$ to lend clarity on the flow field in this high turbulent region. The radial spatial interval of $0.083 D$ is also used in the steady case to obtain the steady spatial velocity profiles.

\subsection{Experimental uncertainty}

The major uncertainties of these experimental measurements are discussed in this section.

The accuracy of hot-wire transverse system is $0.02 \mathrm{~mm}$, resulting in a maximum uncertainty of each travelling distance of $0.4 \%$. The hot-wire anemometer is calibrated every day during the experimental campaign. The offset errors of 


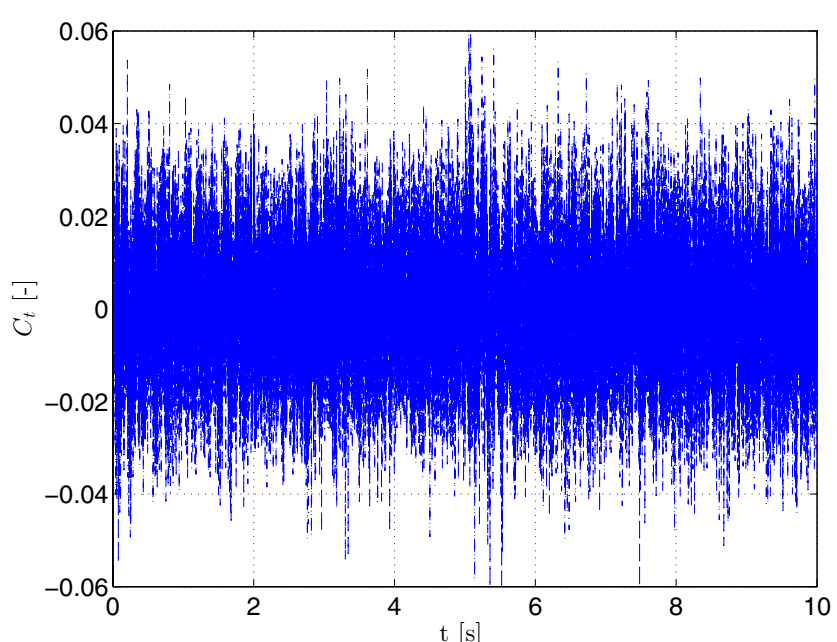

(a) Ensemble average $C_{t}$ at $V_{0}=0$ with actuator on

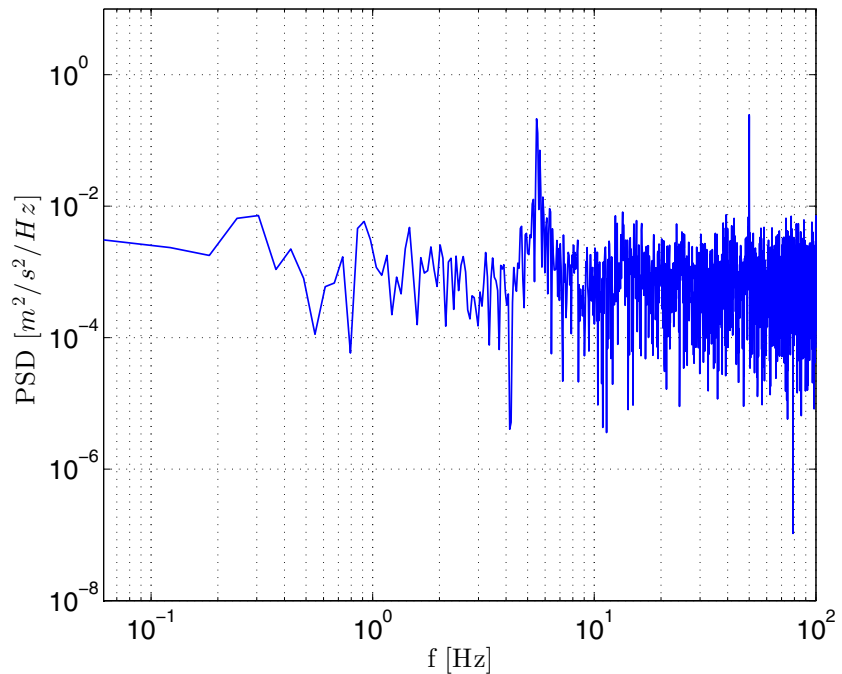

(c) PSD of $C_{t}$ in (a) at $V_{0}=0$ with actuator on

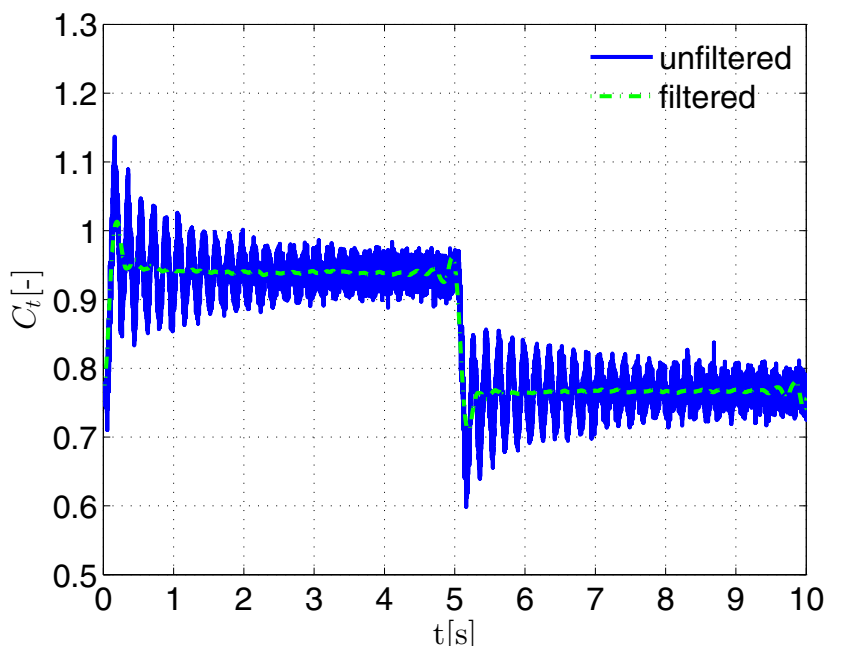

(b) Ensemble average and filtered $C_{t}$ at $V_{0}=6 \mathrm{~m} / \mathrm{s}$ with actuator on

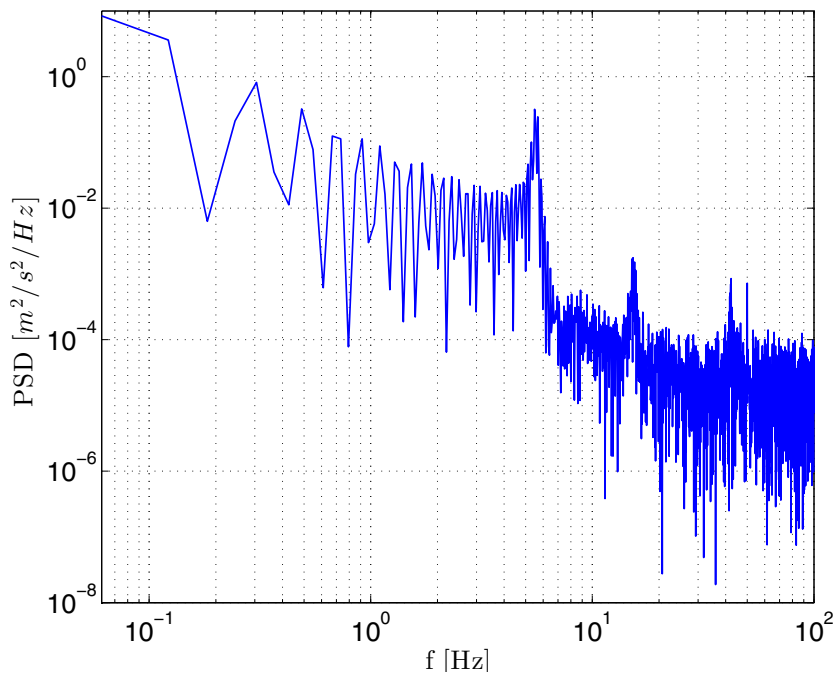

(d) PSD of $C_{t}$ in (b) at $V_{0}=6 \mathrm{~m} / \mathrm{s}$ with actuator on

Fig. $5 C_{\mathrm{t}}$ obtained by the load cell signals with actuator on, at wind speed of 0 or $6 \mathrm{~m} \mathrm{~s}^{-1}$, and their power spectrum

the measured points to the fit curves are kept within $\pm 0.1 \%$ for each calibration.

All the data shown for the unsteady cases are ensemble average values of 10 full cycles. Figure 6 shows the thrust coefficient measured by the load cell and the velocity at location $(x / D=0.5, y / D=0.5)$ in the wake measured by the hot-wire anemometer for case $\delta t^{*}=0.2$, only the first cycle and ensemble average of the first 5 cycles and ensemble average from the 10 cycles are shown. As seen, the more cycles are used, the less noisy will the ensemble average value be. An error of the mean value to numberof-cycles dependency is studied at $(x / D=0.5, y / D=0.5)$, the shear layer region, where the turbulence is most significant. The final 10 cycles is a deliberate value by balancing the accuracy and the needed time, which gives an error less than $0.5 \%$ [more details referred to Hong (2015)]. The natural frequency will be always present as only ensemble average is applied for thrust in Fig. 6 a.

The steady load is determined by both the load cell and the OJF balance after calibrating them with the entire system. Due to the change in temperature during the continuous measurements in the unsteady cases, a drift in both the load cell and the OJF balance signals is observed, a common problem in a load cell. To compensate the effect of load cell drift, the lower mean value of thrust corresponding to the porosity $69.4 \%$ of the unsteady cases is shifted to the measured steady reference value. This process does not have any impact on the velocity and the transient load profiles. 


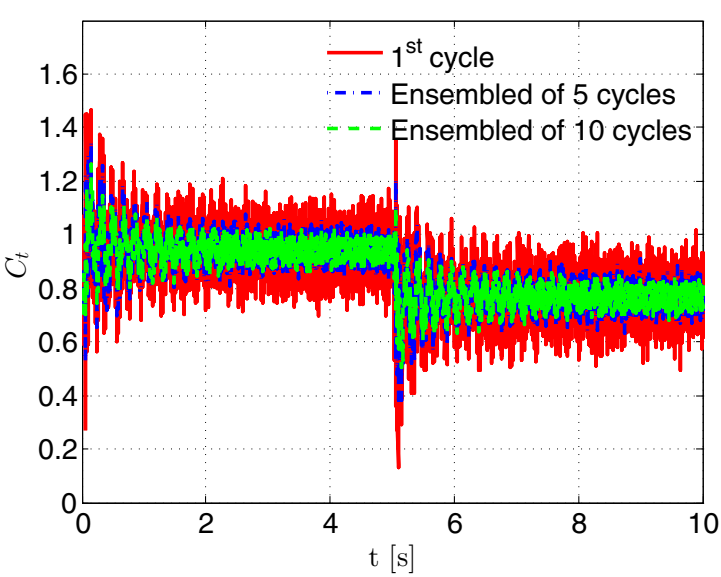

(a) $C_{t}$

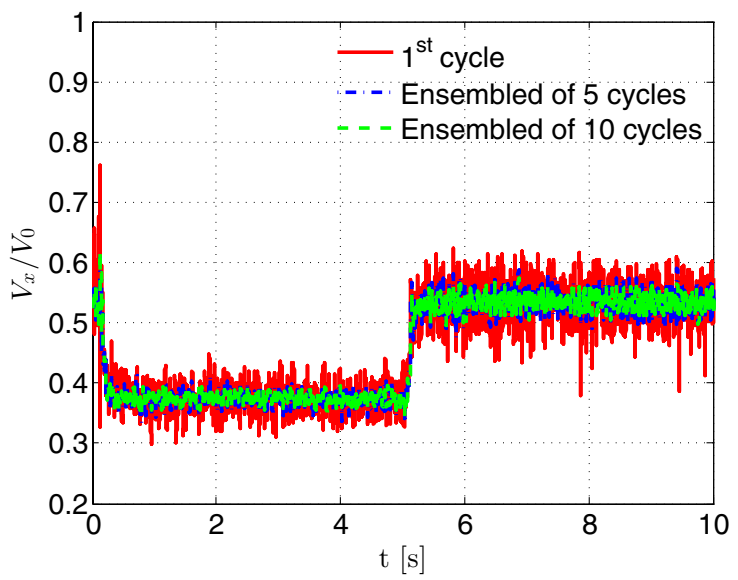

(b) Axial velocity

Fig. 6 Ensemble average thrust and velocity measured at $x / D=0.5, y / D=0.5$ of case $\delta t^{*}=0.2$ for different number of cycles

\section{Results and discussion of steady cases}

\subsection{Disc loading}

From the OJF balance and the load cell signals, the measured time-averaged steady thrust coefficient are $C_{\mathrm{t}}^{\text {low }}=0.767$ and $C_{\mathrm{t}}^{\text {high }}=0.933$, corresponding to porosity values of 69.4 and $44.4 \%$, respectively. The thrust coefficient is defined by

$C_{\mathrm{t}}=\frac{T}{\frac{1}{2} \rho A V_{0}^{2}}$

where $T$ is the measured axial force exerted on the disc by the flow, $A$ is the area of the actuator disc, $V_{0}$ is the velocity of the incoming flow.

\subsection{Velocity measurements}

The time averaged radial profiles of axial velocity and turbulent intensity are plotted in Figs. 7 and 8a, b are a comparison between the downstream planes of each steady case, $\mathrm{c}$ is a comparison between the two steady cases of each downstream plane. From Fig. 7, the velocity in the region around $y / D=0$ is lowest under both steady loads. This is caused by the effect of the nacelle and the tower. As expected, the velocity in the wake for $C_{t}^{\text {low }}$ is larger than that for $C_{t}^{\text {high }}$ for the same locations in Fig. 7c. Additionally, the low porosity state of the disc with $C_{t}^{\text {high }}$ has a higher blockage effect on the velocity outside the wake at $y / D>0.6$. The velocity deficit almost reaches the maximum at $2 D$ for case $C_{\mathrm{t}}^{\text {low }}$ but not even at $3 D$ for the case $C_{\mathrm{t}}^{\text {high }}$, which indicates an earlier starting of wake recovery of a lower thrust. This implies a heavier load has a longer wake recovery distance. From Fig. 7, the axial velocity profiles are not axisymmetric at the hub region. It might be caused by the cable of the load cell, which is bound to the tower only passing one side of the hub (as seen in Fig. 3). The difference decreases for downstream planes and decreases with higher thrust. From Fig. 8, the largest turbulence is observed around $y / D=0$, where the nacelle is. Turbulence decreases as the planes moves downstream from the disc. The second peak is in the shear layer region. Notably, the shear layer of the disc with a lower load persists longer and is relatively stable. The opposite is seen in the higher load case. This can be explained by the higher turbulence intensity and the large pressure gradient in the shear layer of the higher disc load case, which results in an earlier onset of the shear layer breakdown.

\subsection{Disc loads analysis by momentum balance}

Figure 9 illustrates the control volume of the flow used in this axi-symmetric load case for $C_{\mathrm{t}}^{\text {high }}$, being the stream-tube passing through the actuator disc. The measured axial velocities in the wake field are also plotted by the vector arrows. The radii of the wake estimated from the measured velocity are also marked by the red dot on the stream-tube surface, it is approximated at the radial position where the axial velocity is $99 \%$ of the free stream value. The contribution of the lateral force on the stream-tube boundary is zero (Thoma 1925), the axial momentum balance gives

$T=-\left[\rho \int_{A_{\mathrm{w}}} V_{\mathrm{w}}\left(V_{\mathrm{w}}-V_{0}\right) \mathrm{d} A_{\mathrm{w}}+\int_{A_{\mathrm{w}}}\left(p_{\mathrm{w}}-p_{0}\right) \mathrm{d} A_{\mathrm{w}}\right]$

where the thrust on the disc $(T)$ equals but with opposite sign to the thrust exerted on the flow by the disc, $V_{0}$ and $V_{\mathrm{w}}$ represents the velocity of the incoming flow and the velocity in the wake, $p_{0}$ and $p_{\mathrm{w}}$ represents the undisturbed pressure and pressure in the wake, the $A_{\mathrm{w}}$ stands for the area of the stream-tube section in the wake where is examined. 


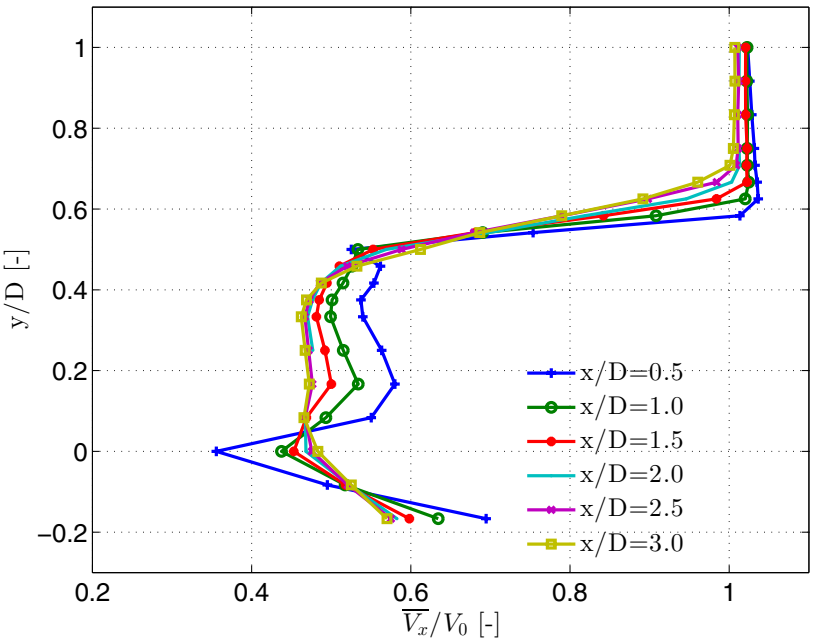

(a) $C_{\mathrm{t}}^{\text {low }}=0.767$

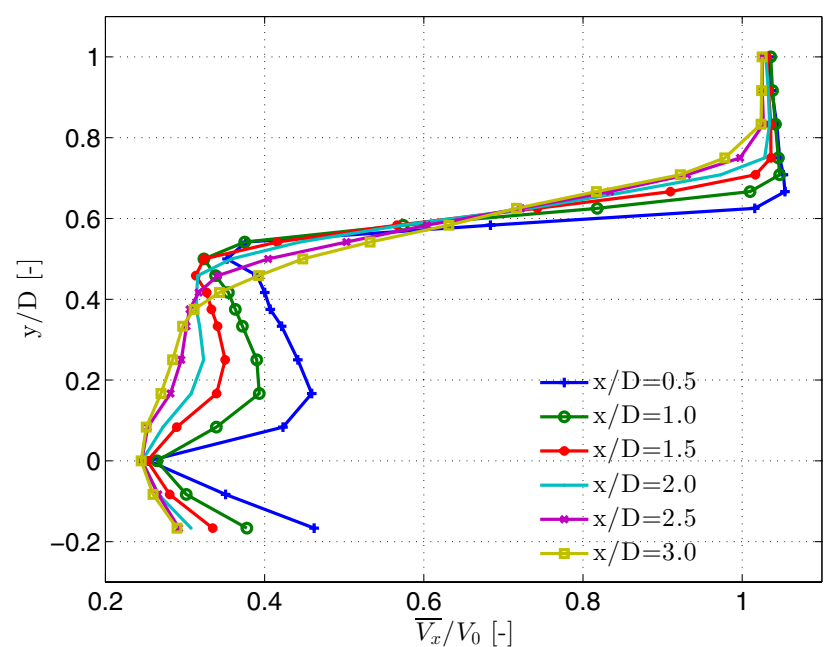

(b) $C_{\mathrm{t}}^{\text {high }}=0.933$

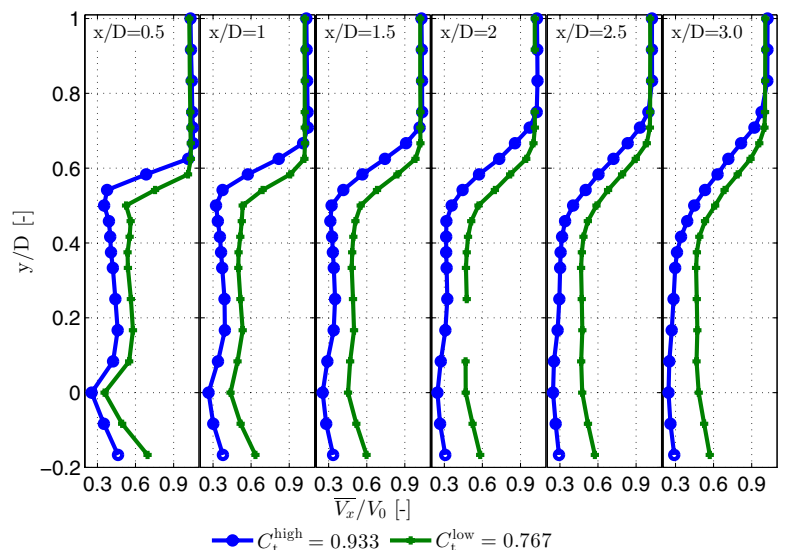

(c) $C_{\mathrm{t}}^{\text {low }}=0.767$ and $C_{\mathrm{t}}^{\text {high }}=0.933$

Fig. 7 Normalized time-averaged axial velocity at different downstream locations under steady loads

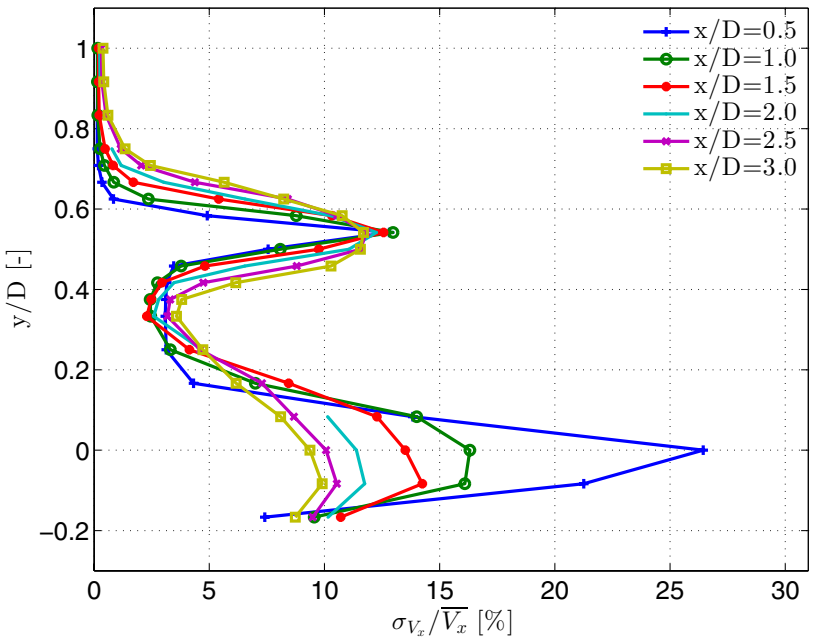

(a) $C_{\mathrm{t}}^{\text {low }}=0.767$

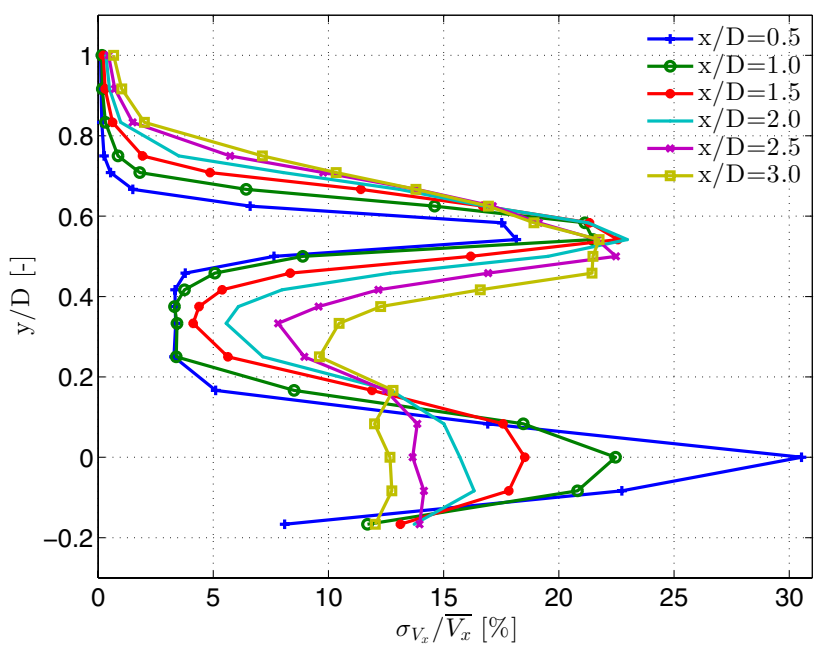

(b) $C_{\mathrm{t}}^{\text {high }}=0.933$

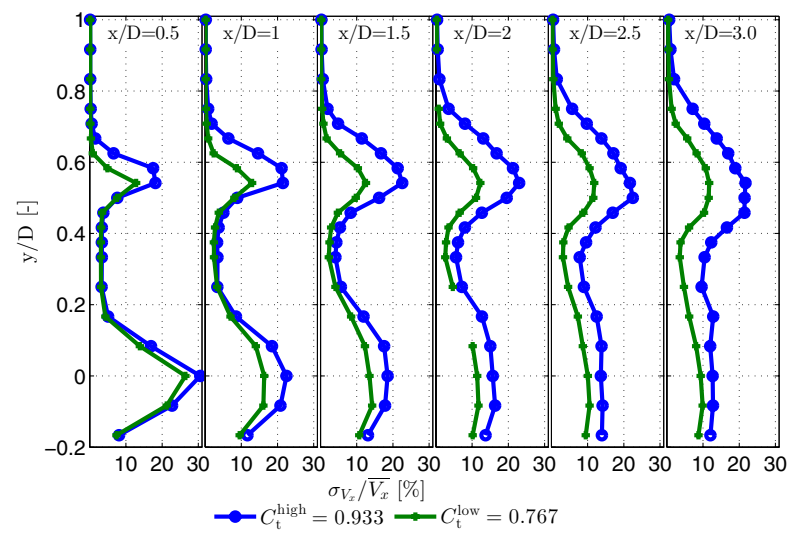

(c) $C_{\mathrm{t}}^{\text {low }}=0.767$ and $C_{\mathrm{t}}^{\text {high }}=0.933$

Fig. 8 Turbulent intensity of axial velocity at different downstream locations under steady loads 


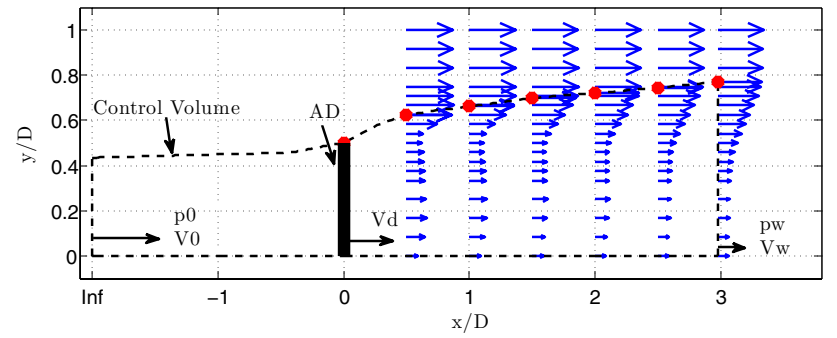

Fig. 9 Control volume of the momentum balance analysis for $C_{\mathrm{t}}^{\text {high }}=0.933$

Table 3 Comparison of thrust coefficient $C_{\mathrm{t}}$ obtained with the direct measurement and calculated from the momentum balance analysis

\begin{tabular}{lll}
\hline Load cases & High load & Low load \\
\hline$C_{\mathrm{t}}$ from measurement & 0.933 & 0.767 \\
$C_{\mathrm{t}}$ from momentum balance & 0.856 & 0.741 \\
$\quad$ analysis & & \\
\hline
\end{tabular}

Under the assumption that the wake is fully expanded at the measured furthest plane $x / D=3.0$, the term $\int_{A_{\mathrm{w}}}\left(p_{\mathrm{w}}-p_{0}\right) \mathrm{d} A_{\mathrm{w}}$ goes to zero in Eq. 4 . The thrust coefficient can be calculated by Eqs. 4 and 3, yielding $C_{\mathrm{t}}=0.856$ for the high load case. The same procedure of momentum balance analysis is applied to the low load case, where $C_{\mathrm{t}}=0.741$ is obtained. The thrust coefficient $C_{\mathrm{t}}^{\text {low }}$ and $C_{\mathrm{t}}^{\text {high }}$ from the directly measured data are $3.4 \%$ and $8.3 \%$ higher than the values calculated from the momentum balance analysis. The comparison is summarized in Table 3. The relative higher values from the experiments might be due to the assumption of $\int_{A_{\mathrm{w}}}\left(p_{\mathrm{w}}-p_{0}\right) \mathrm{d} A_{\mathrm{w}}=0$ at the plane $x / D=3.0$, it can be also caused by the dissipation. As shown in Fig. 7, a heavier load has a longer wake recovery distance, which explains that the relative difference is larger in the case $C_{\mathrm{t}}^{\text {high }}$ than the case $C_{\mathrm{t}}^{\text {low }}$.

\section{Results and discussion of unsteady cases}

In this section, the measured disc displacement and disc loads are presented. The velocity at different locations of the case $\delta t^{*}=0.2$ is discussed. Subsequently, the velocities at different downstream locations between the three different unsteady load cases $\delta t^{*}=0.2,0.4,0.8$ are compared.

\subsection{Disc displacement}

Signals from the encoder of the motor are repeatable during each test case. Figure 10 shows the ensemble average disc displacements measured by the encoder, the view of the time

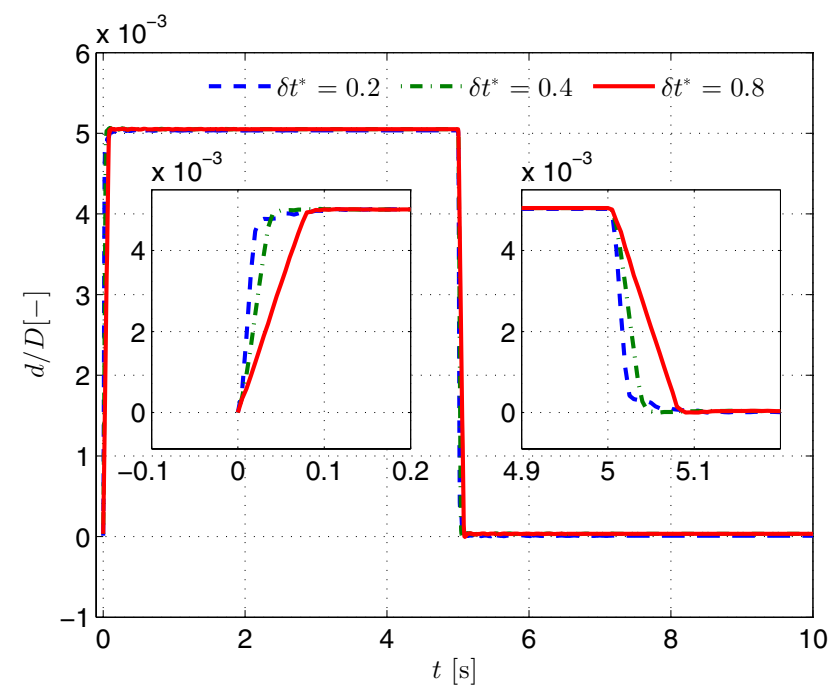

Fig. 10 Ensemble average disc displacement for case $\delta t^{*}=0.2,0.4,0.8$

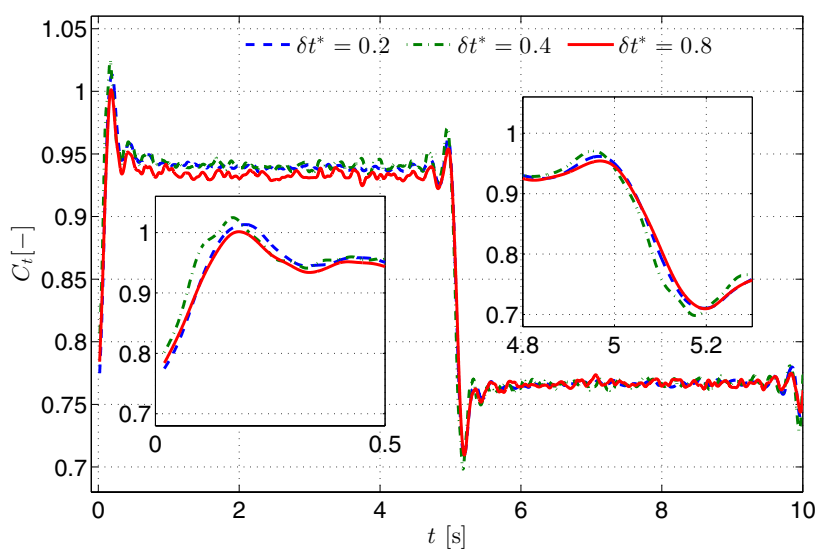

Fig. 11 Ensemble average of the filtered $C_{\mathrm{t}}$ measured by load cell for cases $\delta t^{*}=0.2,0.4,0.8$

period of load increase and decrease are enlarged. It can be seen that the disc displacement is realized according to the design for the two cases of $\delta t^{*}=0.4$ and $\delta t^{*}=0.8$. For the fastest test case $\delta t^{*}=0.2$, the acceleration decreased upon approaching the new steady state due to limited power generated by the actuator.

\subsection{Disc loading}

Figure 11 gives the ensemble average of the filtered thrust coefficient for the three cases measured by the load cell. As seen, there is an overshoot and undershoot in the measured thrust when load increases and decreases. Consistent with references (Carpenter and Fridovich 1953; Pierides et al. 2013), a larger reduced ramp time results in a lower thrust overshoot or undershoot. The relatively small amplitude of 
undershoot and overshoot for the case $\delta t^{*}=0.2$ compared with case $\delta t^{*}=0.4$ is caused by the decreased acceleration when the moving disc reaches the new position as shown in Fig. 10. The thrust starts to respond before the starting time of porosity change is caused by the filter, which can be proved by Fig.5b.

\subsection{Velocity profile for unsteady case $\delta t^{*}=0.2$}

The velocity at a number of locations inside and outside the wake is measured (as shown in Fig. 1), but only three main radial positions which can highlight the characteristics of the flow aft of the disc are presented. The three radial locations are at (a) the inner wake, $y / D=0.33$, (b) the shear layer, $y / D=0.58$ and (c) the outer wake, $y / D=0.83$. The ensemble average velocity for the six downstream planes $x / D=0.5,1.0,1.5,2.0,2.5,3.0$ at the three radial locations are plotted in Fig. 12. The mean flow characteristics under steady conditions (i.e. between $3-5$ and $8-10 \mathrm{~s}$ of the unsteady cycles) have been discussed in the Sect. 3.2, the discussion here will focus on the transient behaviour during the load change.

As seen from Fig. 12, the flow in the shear layer region $y / D=0.58$ is most turbulent due to the large vorticity in this region. However, due to the smaller order of turbulence when compared to the jump in velocity, the transient changes of flow are still discernible. As expected, the flow in the outer wake $y / D=0.83$ is the least turbulent because the flow in this region has not passed the disc. Resulting from the effect of holes in the porous discs and the shadow of nacelle and tower, the flow in the inner wake $y / D=0.33$ is relatively more turbulent than that in the outer region.

Considering the same point in the outer wake region, for example $(x / D=3.0, y / D=0.83)$, the flow is more turbulent
Fig. 12 Ensem-

ble average velocity at $y / D=0.33,0.58,0.83$ for downstream planes $x / D=0.5,1.0,1.5,2.0,2.5,3.0$ under unsteady loads for case $\delta t^{*}=0.2$
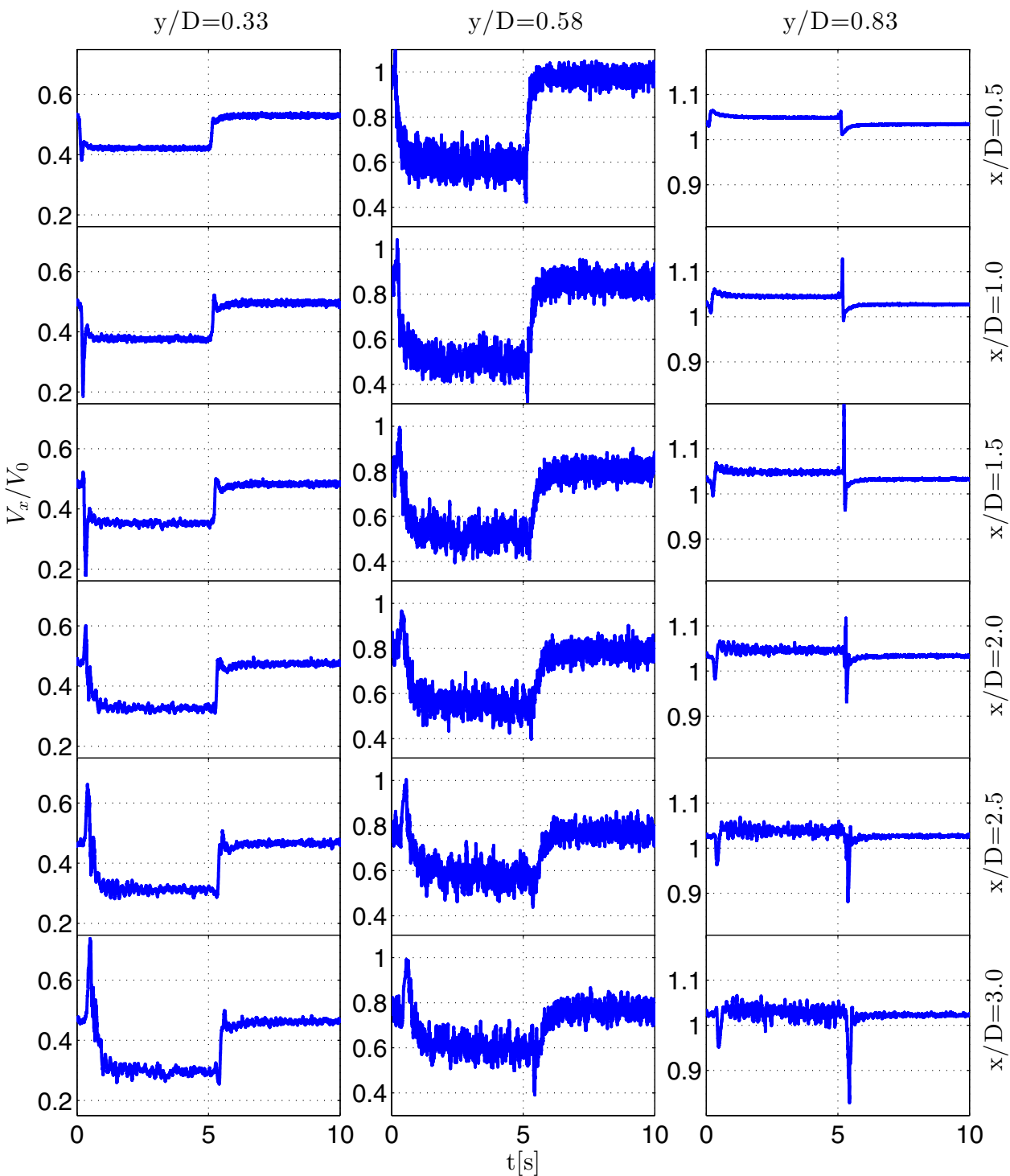
for the high thrust than that for the low thrust. This shows the effect of wake expansion, the point $(x / D=3.0, y / D=0.83)$ is outside the shear layer at the low thrust $C_{\mathrm{t}}^{\text {low }}$, but closer to the shear layer at the high thrust $C_{\mathrm{t}}^{\text {high }}$ when the wake expansion is larger.

The key observation is the overshoot or undershoot in the flow velocity before it decays to the steady state. The overshoot or undershoot was only observed in load measurement in previous projects (Snel and Schepers 1995; Schepers and Snel 1995; Hand et al. 2001), where it was explained by the cause of unsteady airfoil aerodynamics. Airfoil aerodynamics do not play a role in this experiment with disc, it might provide clues on the cause of overshoot or undershoot in thrust. In the inner wake $y / D=0.33$ and shear layer region $y / D=0.58$, the amplitude of overshoot or undershoot is larger for load increase than for load decrease. On the contrary, in the outer wake $y / D=0.83$, the amplitude of overshoot or undershoot is larger for load decrease than for load increase. It is hypothesised that the velocity overshoot or undershoot is caused by the passage of the shed vorticity at the disc edge, which is generated when the thrust changed abruptly. The fact that measurements show the overshoot or undershoot in velocity having a delay proportionate to the downstream distance, suggests a travelling and momentary 'one-time' phenomena that has propagated from the disc upon the sudden load change. This implies the passage of the vorticity. The physical cause of the velocity overshoot or undershoot and the difference at different locations will be further studied in the near future.

\subsection{Comparison of velocity profile for three unsteady cases}

This section compares the measured velocity profiles in the wake between the three different cases $\delta t^{*}=0.2,0.4,0.8$. Figure 13 compares the velocity of the three cases at the radial position $y / D=0.33,0.83$ of the downstream planes $x / D=1.0,2.0$ and 3.0, after load increase and decrease, respectively.

First of all, what is observed in Sect. 4.3 still holds for all the three cases in Fig. 13. Second, although the velocity profiles between the three cases are similar, the effect of speed of load change, the reduced ramp time, can be seen. It can be generally concluded that the smaller the reduced ramp time (the more rapid the load change), the larger the velocity overshoot or undershoot, and the steeper the slope of the transient velocity profiles.

The ensemble average velocity at the radial locations $y / D=0,0.17,0.33,0.42,0.58,0.83$ of the plane $x / D=1.0$ are shown in Fig. 14 for load increase and decrease, respectively. What is already discussed in Fig. 13 is also relevant in Fig. 14, the speed of load change mainly affects the initial velocity transient profiles. A steeper ramp time results in a faster velocity transient, and in turn a larger amplitude of velocity overshoot or undershoot. The difference between the three cases is less distinct near the region around $y / D=0$ where the wake effect of the nacelle and tower dominates, and the shear layer region $y / D=0.58$ where the flow is susceptible to the higher turbulence.

The overshoot or undershoot appears intermittently at different radial locations for the same plane. For instance, in the load increase case in Fig. 14, there is an undershoot in the velocity at the locations $y / D=0.17-0.42$, but an overshoot at locations $y / D=0.5-0.58$. This might be caused by their

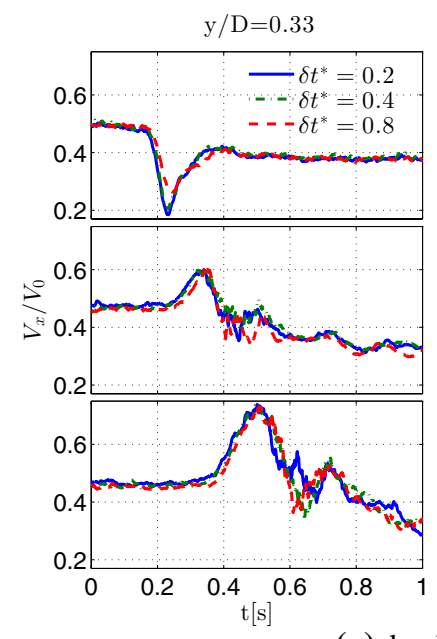

(a) load increase

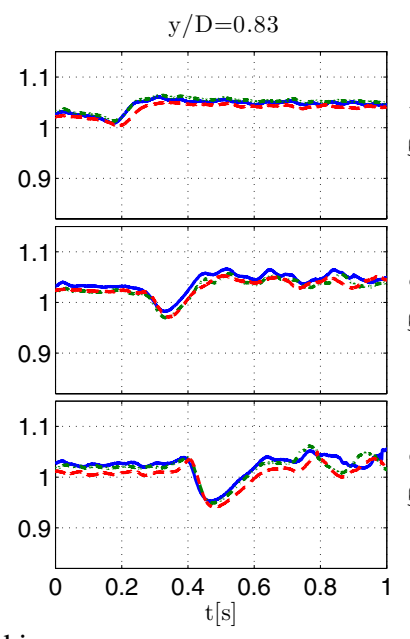

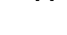
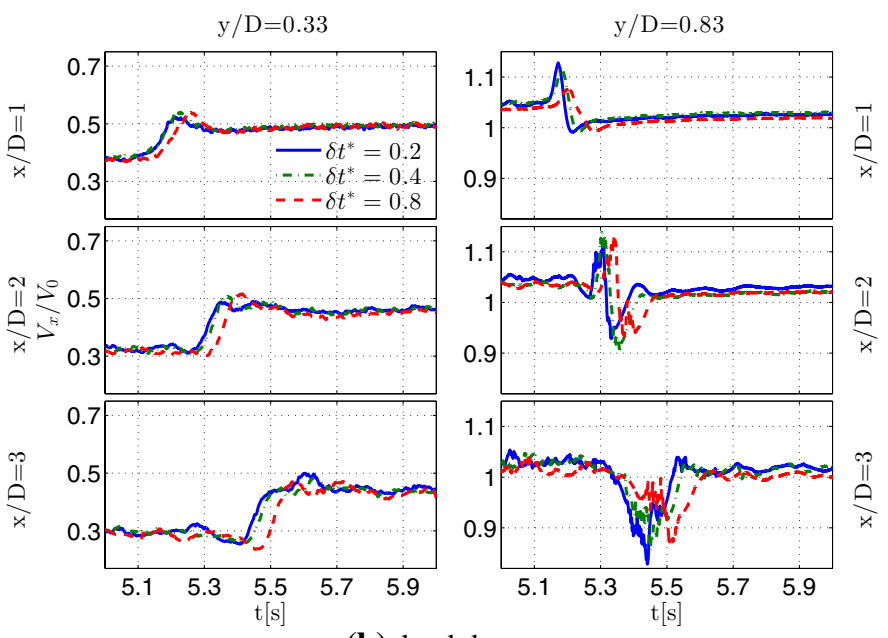

(b) load decrease

Fig. 13 Ensemble average velocity at different downstream locations under unsteady loads for case $\delta t^{*}=0.2,0.4,0.8$ 
Fig. 14 Ensem-

ble average velocity at $y / D=0,0.17,0.33,0.42,0.5$, $0.67,0.83$ for downstream planes $x / D=1.0$ under unsteady loads for cases $\delta t^{*}=0.2,0.4,0.8$

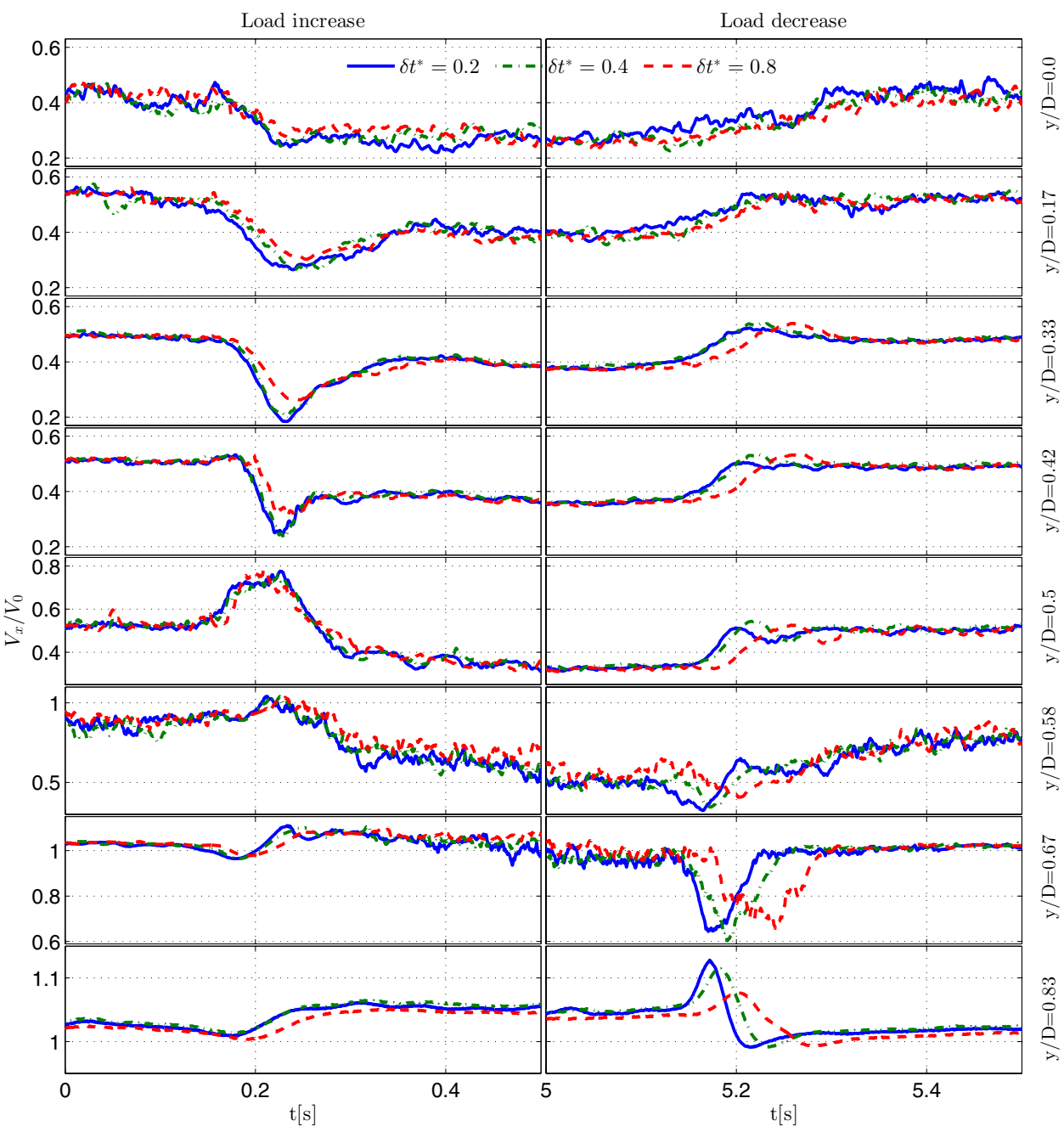

different relative positions to the convecting vortex, which is shed at the disc edge when the thrust suddenly changed.

The other observation is that the onset time of flow responding to the disturbance from the disc varies between different radial locations of the same plane, which implies that the convection velocity of disturbance varies radially. Additionally, it can be seen that the time the velocity takes to transition from the old steady state to the new steady state also varies radially. The phenomena indicates that the time constant of flow decay in the wake field varies in the radial direction, which will be further discussed in Sect. 4.5.

\subsection{Time constant for dynamic inflow decay}

The time constant of the measured velocity decay is not straightforward due to the velocity fluctuations. The similar approach used in Snel and Schepers (1995) is used, where the velocity profile is approached by an exponential behaviour according to the following equation

$V(t)=V_{1}+\Delta V *\left(1-\exp ^{-\frac{t-t_{1}}{\tau}}\right)$
The determination of $V_{1}, t_{1}, \Delta V$ are given in Fig. 15 as an example for two typical different situations of measured wake velocity. For most locations, there is a velocity overshoot, where the peak value and the according peak time are treated as the starting value $V_{1}$ and the starting time $t_{1}$ of the decay. For the situation where there is no velocity overshoot, the last value before abrupt change is treated as the starting value $V_{1}$ and the according time is treated as the starting time $t_{1}$.

The mean value of the velocity from $2 \mathrm{~s}$ after load change to the new load change start (i.e. between 3-5 and 8-10 s of the unsteady cycle) is treated as the new steady value $V_{2}$. Consequently, $\Delta V=V_{2}-V_{1}$.

The fitted curves are also plotted in Fig. 15.

The dynamic inflow time constants for velocity at all the measured locations are plotted separately for load increase and decrease cases, for the three reduced time cases in Fig. 16. The following general observations can be drawn from the comparison:

The decay time outside the wake is generally larger than that inside the wake for a given downstream plane. The 


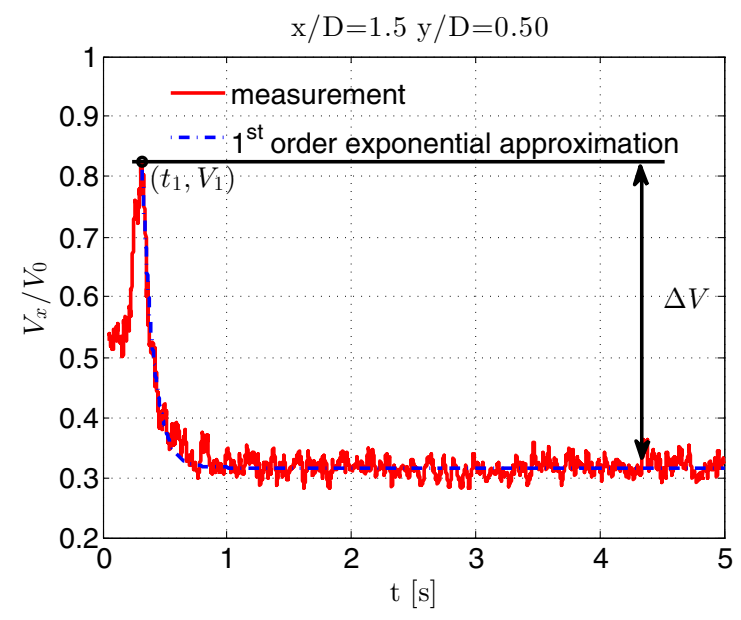

(a) case with overshoot

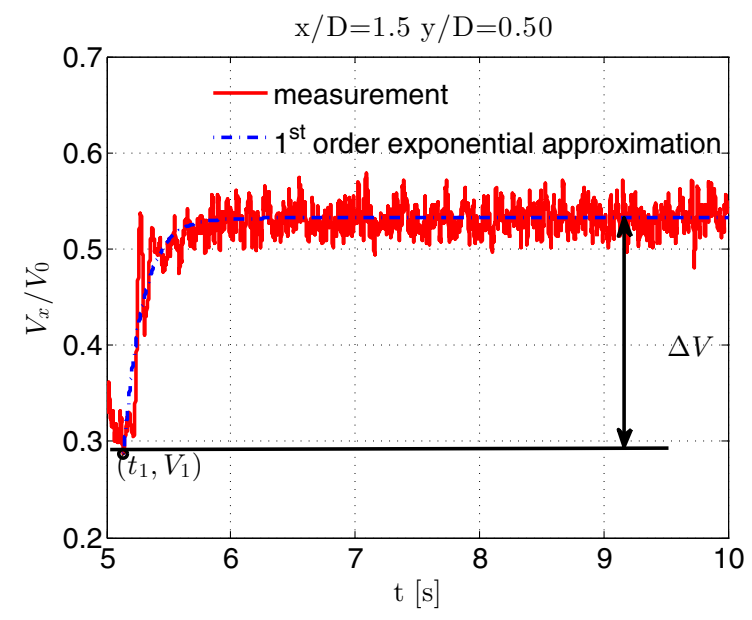

(b) case without overshoot

Fig. 15 Measured velocity and one order exponential approximation to determine the dynamic inflow time constant

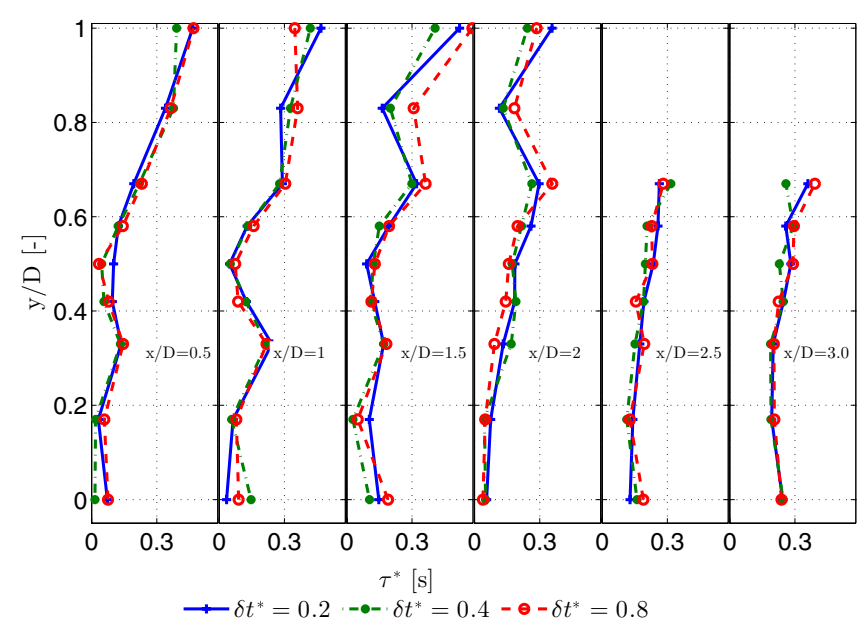

(a) load increase

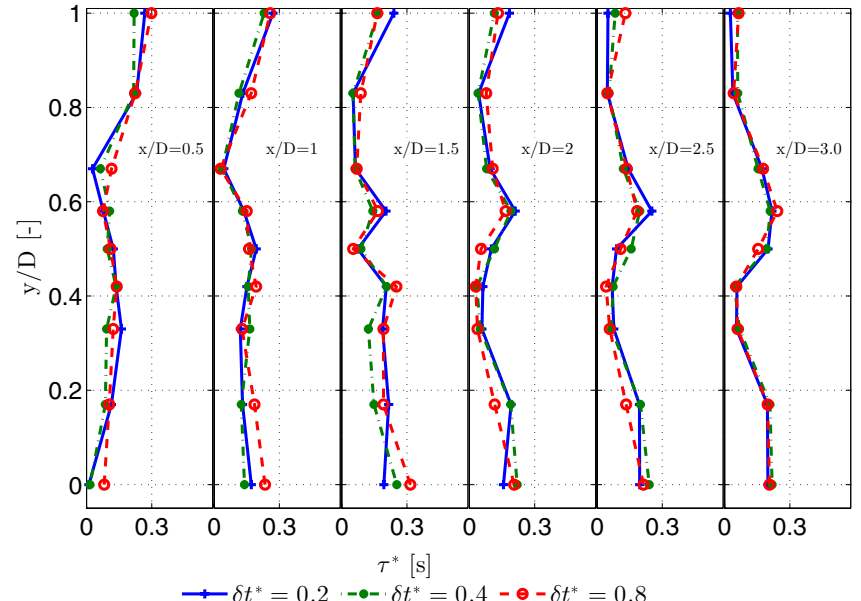

(b) load decrease

Fig. 16 The dynamic inflow time constant estimated from the measured velocity at different locations for cases $\delta t^{*}=0.2,0.4,0.8$

shorter decay time might be caused by the relative higher turbulence intensity inside the wake, which facilitates momentum mixing and results in the faster attainment of the new equilibrium.

For the same locations, the decay time for load increase is relatively larger than for load decrease. This observation is consistent with the trend of induction change at the actuator disc in Yu et al. (2016), where the induction at the actuator disc reaches the new steady values faster in the load decrease case than in the load increase case. This is because the larger disc load results in a larger induction field, which gives a slower convection velocity of the new generated vorticity. This leads to a slower equilibrium of the new steady state.

The time constants is not uniform for different radial positions of the same plane.
The time constants of velocity at $y / D=0-0.17$ have been influenced by the hub and tower evidently. For the near downstream planes $x / D=0.5$, the time constants decrease from $y / D=0.33$ towards to $y / D=0.5$ for the load increase cases, decrease from $y / D=0.33$ towards to $y / D=0.67$ for the load decrease cases. This direct wake measurement is an important evidence for the same finding by an analytical model in Schepers (2007) that the time constants at the actuator disc plane reduce towards to the edge of the disc. However, Schepers (2007) also showed this finding is not affirmed by the previous load data measured from the NASA Ames Phase VI unsteady aerodynamic experiments (Hand et al. 2001) and numerical simulations from AWSM (Van Garrel 2003), a free wake vortex code. 


\section{Interpretation of the results and conclusions}

In this paper, the flow at locations downstream of an unsteady actuator disc is studied by a hot-wire anemometer. The unsteady load of the actuator disc is invoked by changing the porosity of the actuator formed by two identical metal screens. Flow is measured for three different change rates of porosity.

\subsection{Interpretation of the results}

\subsubsection{Results to be expected}

The velocity and turbulence profiles are shown to be characterised by three regions, the inner wake region, the shear layer region and the region outside the wake.

Due to different distances from the disc, the flow at different downstream planes responds to the disturbance exerted by the disc successively.

The flow reaches the new steady state slower in the load increase case than the load decrease case due to the larger induction in the wake field of the load increase case.

\subsubsection{New findings}

The time constants outside the wake are generally larger than that inside the wake for a given downstream plane due to the relative higher turbulence intensity inside the wake.

In addition to the transient change of velocity to the new steady state, an overshoot or undershoot in velocity is observed at different locations in the field. A difference in overshoot or undershoot is observed between cases when loading is increased and loading is decreased, also between the different locations in the field where the velocity is measured. The velocity overshoot or undershoot is hypothesised to be caused by the passage of the shed vorticity at the disc edge, which is generated when the thrust changed abruptly.

The reduced ramp time is found to have an impact on the wake dynamics, the smaller the reduced ramp time (the faster the load change), the steeper the transient velocity change is and the larger the amplitude of the velocity overshoot or undershoot.

The time constants at the same plane show a radial dependence.

\subsection{Conclusions}

From the observations and the interpretation of the results, it can be concluded that the convection velocity of the wake and the local turbulence intensity are the key factors determining the total transient time from one steady state to another steady state. The impact of turbulence intensity on the transient time needs to be further quantified for the future work. The shed vorticity plays an important role in the dynamic inflow problem, it can determine the overshoot and undershoot of velocity in the wake, the radial dependence of time constants. Better understanding of this by detailed unsteady wake study using numerical and experimental methods is of interest for the future work.

Acknowledgements The first author gratefully acknowledges financial support from China Scholarship Council. The great help from Daniel Baldachinno, Lorenzo Lignardo, Daniele Ragni and Francesco Avallone for the final success of the experiments is highly acknowledged. Bruce LeBlanc is thanked for help with the improvement of English of this paper.

Open Access This article is distributed under the terms of the Creative Commons Attribution 4.0 International License (http://creativecommons.org/licenses/by/4.0/), which permits unrestricted use, distribution, and reproduction in any medium, provided you give appropriate credit to the original author(s) and the source, provide a link to the Creative Commons license, and indicate if changes were made.

\section{Appendix: Supplementary data}

Supplementary data related to this article can be found at https://data.4tu.nl/repository/ uuid:c2000ce7-3c56-45e4-a55d-22b33060d289.

\section{References}

Aubrun S, Loyer S, Espana G, Hayden P, Hancock P (2011) Experimental study on the wind turbine wake meandering with the help of a non-rotating simplified model and of a rotating model. In: 49th AIAA aerospace sciences meeting including the new horizons forum and aerospace exposition, Florida, January, pp 1-6. doi:10.2514/6.2011-460

Aubrun S, Loyer S, Hancock PE, Hayden P (2013) Wind turbine wake properties: comparison between a non-rotating simplified wind turbine model and a rotating model. J Wind Eng Ind Aerodyn 120:1-8. doi:10.1016/j.jweia.2013.06.007

Bossuyt J, Howland M, Meneveau C, Meyers J (2017) Measurement of unsteady loading and power output variability in a micro wind farm model in a wind tunnel. Exp Fluids 58(1):1. doi:10.1007/ s00348-016-2278-6

Camp E, Cal R (2016) Mean kinetic energy transport and event classification in a model wind turbine array versus an array of porous disks: energy budget and octant analysis. Phys Rev Fluids 1(4):044404. doi:10.1103/PhysRevFluids.1.044404

Carpenter P, Fridovich B (1953) Effect of a rapid blade-pitch increase on the thrust and induced-velocity response of a full-scale helicopter rotor. Tech. Rep, November, National Advisory Committee for Aeronautics, Washington

Castro I (1971) Wake characteristics of two-dimensional perforated plates normal to an air-stream. J Fluid Mech 46:599-609

Chamorro L, Porté-Agel F (2009) A wind-tunnel investigation of windturbine wakes: boundary-layer turbulence effects. Bound Layer Meteorol 132(1):129-149. doi:10.1007/s10546-009-9380-8

Froude RE (1889) On the part played in propulsion by differences of fluid pressure. Trans Inst Naval Archit 30:390-405 
Hand M, Simms D, Fingersh L, Jager D, Cotrell J, Schreck S, Larwood S (2001) Unsteady aerodynamics experiment phase VI: wind tunnel test configurations and available data campaigns. Tech. Rep, December, National Renewable Energy Laboratory, Colorado

Hoerner SF (1965) Fluid-dynamic drag: theoretical, experimental and statistical information. Hoerner fluid dynamics, New York

Hong VW (2015) Analysis of an actuator disc under unsteady loading. Ph.D. thesis, Delft University of Technology

Johnson P, Wojcik A, Drake K, Eames I (2013) Impulsively started planar actuator surfaces in high-Reynolds-number steady flow. $\mathbf{J}$ Fluid Mechan 733:302-324. doi:10.1017/jfm.2013.427

Knight M (1926) Technical note NO. 253.-Wind tunnel standardization disk drag. Tech. rep., National Advisory Committee for Aeronautics, Langley Aeronautical Laboratory, Washington

Lignarolo L, Ragni D, Ferreira C, van Bussel G (2014) Kinetic energy entrainment in wind turbine and actuator disc wakes: an experimental analysis. J Phys Conf Ser 524(Torque):1-10. doi:10.1088/1742-6596/524/1/012163

Lignarolo L, Ragni D, Ferreira C, van Bussel G (2016) Experimental comparison of a wind-turbine and of an actuator-disc near wake. J Renew Sustain Energy 8:1-27. doi:10.1063/1.4941926

Medici D (2005) Experimental studies of wind turbine wakes-power optimisation and meandering. Ph.D. thesis, KTH Mechanics

Muller YA, Aubrun S, Masson C (2015) Determination of real-time predictors of the wind turbine wake meandering. Exp Fluids 56(3):1-11. doi:10.1007/s00348-015-1923-9

Øye S (1986) Unsteady wake effects caused by pitch-angle changes. In: Proceedings of the first IEA symposium on the aerodynamics of wind turbines, London

Øye S (1990) A simple vortex model of a turbine rotor. In: Proceedings of the third IEA symposium on the aerodynamics of wind turbines, Harwell, pp 1-15

Øye S (1991a) Tjæreborg wind turbine, 4. dynamic inflow measurement. Tech. rep., Technical University of Denmark, Lyngby
Øye S (1991b) Tjæreborg wind turbine (Esbjerg): First dynamic inflow measurement. Tech. Rep. VK-189, Technical University of Denmark, Lyngby

Pierella F, Sætran L (2010) Effect of initial conditions on flow past grids of finite extension. In: 17th Australasian fluid mechanics conference, Auckland, December

Pierides A, Elzawawy A, Andreopoulos Y (2013) Transient force generation during impulsive rotation of wall-mounted panels. J Fluid Mech 721:403-437. doi:10.1017/jfm.2013.52

Pitt D, Peters D (1981) Theoretical prediction of dynamic-inflow derivatives. Vertica 5(1):21-34

Schepers J (2007) IEA Annex XX : dynamic inflow effects at fast pitching steps on a wind turbine placed in the NASA-Ames wind tunnel. Tech. rep, ECN, Petten

Schepers J (2012) Engineering models in wind energy aerodynamics. Ph.D. thesis, Delft University of Technology

Schepers J, Snel H (1995) Dynamic inflow: yawed conditions and partial span pitch control. Tech. rep, ECN, Petten

Sforza P, Sheerin P, Smorto M (1981) Three-dimensional wakes of simulated wind turbines. AIAA J 19(9):1101-1107

Snel H, Schepers J (1995) Joint investigation of dynamic inflow effects and implemention of an engineering method. Tech. rep, ECN

Thoma D (1925) Grundsatzliches zur einfachen Strahltheorie der Schraube. Zeitschrift fr Flugtechnik und Motorluftschiffahrt 16:206-208

Van Garrel A (2003) Development of a wind turbine aerodynamics simulation module. Tech. rep, ECN

Yu W, Ferreira C, van Kuik G, Baldacchino D (2016) Verifying the blade element momentum method in unsteady, radially varied, axisymmetric loading using a vortex ring model. Wind Energy. doi:10.1002/we2005 